\title{
Speech-induced lingual dystonia
}

\author{
Andre C. Felicio ${ }^{1,2}$, Clecio Godeiro-Junior' ${ }^{1}$, Tais S. Moriyama2,3, \\ Maura R. Laureano², Evandro P. V. Felix', Vanderci Borges', \\ Sonia M. A. Silva', Henrique B. Ferraz'
}

\begin{abstract}
Dystonia can be classified according to its etiology as primary, dystonia-plus, secondary, heredodegenerative, and psychogenic $^{1}$. Pure lingual dystonia is rare but not uncommon in association with other cranial dystonia. However, in clinical practice, even after excluding the main secondary causes of dystonia, the differential diagnosis between primary versus psychogenic forms may be tricky ${ }^{1}$. There are few cases in the literature describing speech-induced lingual dystonia and they were mostly classified as idiopathic ${ }^{2-5}$. We report a case of a woman who presented with a speech-induced lingual dystonia and discuss the differential diagnosis and potential therapeutic options of this condition.
\end{abstract}

\section{CASE}

A 49-year-old white woman was referred to our service with a six-year history of tongue protrusion when speaking. There was no relevant past or family history of neurological disorders. She did not take neuroleptics or other medications before the onset of symptoms and had no history of facial injury or infection. For six years she visited several physicians and no diagnosis was made. On her first appointment at our service she was taking clonazepam $2 \mathrm{mg} /$ day without improvement. Her neurological exam showed speech-induced tongue protrusion associated with mild dysarthria. The movement disorder showed no improvement with chewing gum (sensory trick). The patient could eat, drink, whistle, sing, and whisper without any trouble. A trial with levodopa $(750$ $\mathrm{mg} /$ day) and then trihexyphenidyl (10 mg/ day) did not ameliorate symptoms.
A number of exams were ordered to rule out secondary dystonia. Drug-induced, dopa-responsive, post-traumatic and post-infectious dystonias had already been ruled out, and the absence of family history suggested no heredodegenerative disorder. At this time the diagnoses considered were neuroacanthocytosis, Wilson's disease, and pantothenate kinase-associated neurodegeneration. Routine hematological and biochemical evaluation were completely normal including copper levels and number of acanthocytes. Brain magnetic resonance imaging (MRI), electroencephalography (EEG), and electromyography (EMG) were within normal parameters.

A speech-therapist evaluated the tongue movement during several tasks: repeated words and sentences, reading a short text, automatic speech, singing, vowel and fricative phoneme prolongation, sequences of syllables, and spontaneous conversation. The tongue movement disorder was identified in all circumstances of speech and in all phonemes, except vowel and sound prolongations. Tongue protrusion occurred more often in alveodental and alveolar phonemes and less frequently in palatal and velar phonemes. Slower speech and low voice intensity improved tongue protrusion.

After the initial work-up ruling out many etiologies, we investigated non-organic causes and referred her to a psychiatric examination. In this evaluation the patient told that her symptoms started during a period she went through a serious moral dilemma while working in an illegal informal job she considered humiliating. She also told that these symptoms could
Department of Neurology

Rua Pedro de Toledo 650 04039-002 São Paulo SP - Brasil

E-mail: cf.andre@gmail.com

Received 23 July 2009

Received in final form 11 September 2009 Accepted 24 September 2009

\section{DISTONIA DE LÍNGUA INDUZIDA PELA FALA}

Federal University of São Paulo, São Paulo SP, Brazil: 'Department of Neurology and Neurosurgery; ${ }^{2}$ Interdisciplinary Clinical Neurosciences Laboratory; ${ }^{3}$ Department of Psychiatry. 
be related to psychological causes so that severity tended to increase when she was feeling herself guilt. During the psychiatric appointment the movement disorder improved with external suggestion that consisted of stopping tongue protrusion while speaking, achieving brief periods of full remission (lasting up to 5 minutes). The same improvement was not observed outside the medical office. According to the Structured Clinical Interview for DSM IV, she had the diagnosis of major depressive episode 8 years ago and no other diagnosis.

Although a psychogenic etiology for her dystonia could not be ruled out just because we did not identify sensory trick, positive neuroimaging or laboratory data, and positive neurophysiological studies, or due to the patient's personal problems and stressing events, we considered the etiology as primary. After a six-month follow-up, she still presents the same tongue movement disorder, although without specific medication. We did not use Botulinum Toxin (BTX) type-A injections since the movement disorder did not worsen. A signed-informed consent was obtained from the patient to allow her data publication.

\section{DISCUSSION}

Tongue movement disorders comprise a poorly understood group of diseases, and several clinical presentations were described, such as tremor, myokimia and dyskinesias. However, there are few reports on speech-induced lingual dystonia ${ }^{2-5}$, and generally a primary etiology is considered.

Table shows a review of patients with speech-induced lingual dystonia. Two of the four cases reported in the literature considered a psychogenic etiology for lingual dystonia ${ }^{2,3}$. Interestingly, in both cases, interviews by psychologists and psychometric testing indicated a normal psychiatric state and ruled out a character disorder. In our case, we also considered a primary etiology despite several psychiatric stressors identified and negative work-up.

We made a thorough speech assessment of our patient. The goal was to evaluate any pattern of tongue protrusion dystonia, since no data about this issue was available in the literature. We found that the tongue movement disorder was present in all circumstances of speech and in all phonemes, except vowel and sound prolongations. Therefore, further studies comparing the pattern of speech abnormalities in patients with speech-induced lingual dystonia with healthy control subjects deliberately generating tongue protrusion during speaking would be of interest.

There are other unusual reports of perioral dystonia/ dyskinesias affecting the lower facial muscles and jaw, but these disorders are mainly present at rest and seldom during speech ${ }^{6-8}$. Paroxysmal movement disorders such as paroxysmal kinesogenic dyskinesias and episodic ataxias are well described and usually involve the limbs.

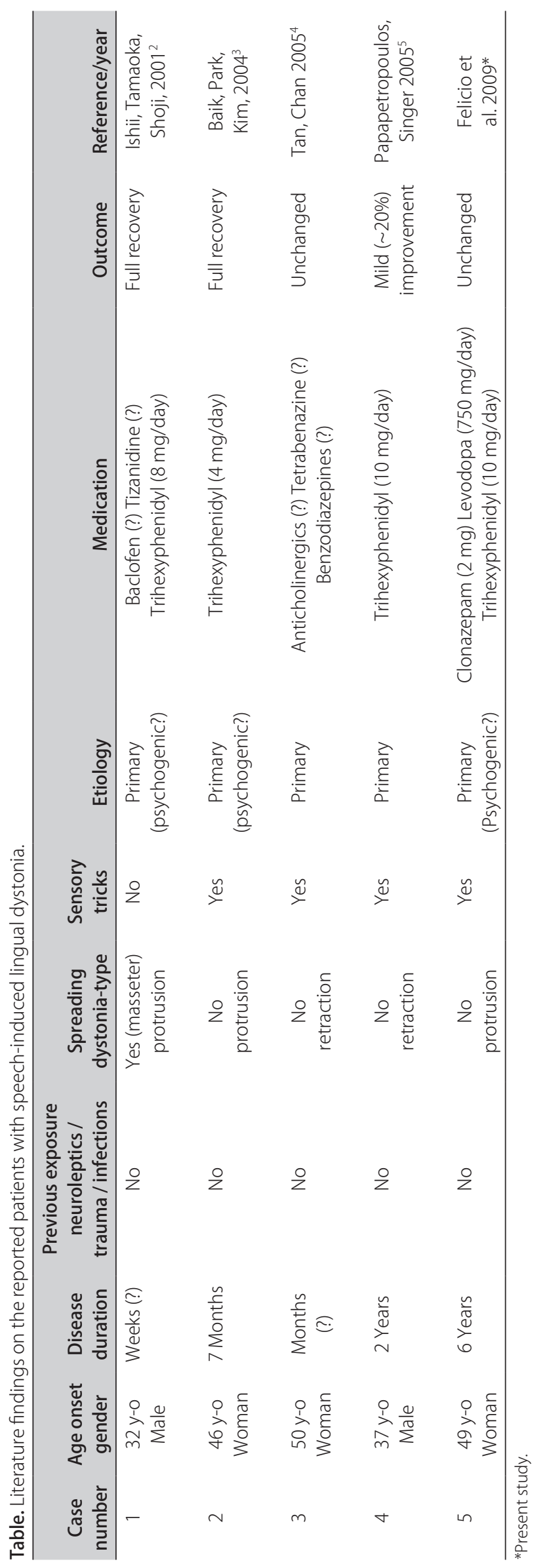


Spasms of the tongue are seldom reported to occur in these syndromes ${ }^{6,7}$. Therefore, episodic or paroxysmal movement disorders involving the tongue although rare should be taken as differential diagnosis for patients with dystonia speech-induced. Focal lingual dystonia or tremor have been associated with trauma, especially with electrical injuries and is another form of exclusive involvement of the tongue 9

The best treatment for the primary forms of oro-buccallingual dystonias is BTX injections ${ }^{10}$. No patients reported with speech-induced lingual dystonia (including ours) were treated with $\mathrm{BTX}^{2-5}$. Actually, anticholinergic agents were the oral medications more often prescribed (Table).

Treatment with BTX type-A injection in the genioglossus in a series of nine patients with involuntary tongue protrusion due to oromandibular dystonia or Meige's syndrome showed marked reduction in tongue protrusion in six patients, suggesting that BTX type-A may be a valid option for involuntary tongue protrusion ${ }^{11}$. Dysphagia is a frequent complication of treating lingual dystonia with BTX and it may lead to choking. Therefore, further studies are needed to better evaluate the efficacy of this BTX in lingual dystonia, minimizing dysphagia, an undesirable side-effect ${ }^{12}$.
In conclusion, speech-induced lingual dystonia should be considered as one of the presentation forms of taskspecific tongue movement disorders and physicians should be aware of its differential diagnosis.

\section{REFERENCES}

1. Langlois M, Richer F, Chouinard S. New perspectives on dystonia. Can J Neurol Sci 2003;30:34-44

2. Ishii K, Tamaoka A, Shoji S. A case of primary focal lingual dystonia induced by speaking. Eur J Neurol 2001;8:507.

3. Baik JS, Park JH, Kim JY. Primary lingual dystonia induced by speaking. Mov Disord 2004;19:1251-1252.

4. Tan EK, Chan LL. Sensory tricks and treatment in primary lingual dystonia. Mov Disord 2005;20:388

5. Papapetropoulos $S$, Singer C. Primary focal lingual dystonia. Mov Disord 2006;21:429-430.

6. Edwards M, Schott G, Bhatia K. Episodic focal lingual dystonic spasms. Mov Disord 2003;18:836-837.

7. Lees AJ, Blau JN, Schon F. Paroxysmal hemiglossal twisting. Lancet 1986;2: 812-813.

8. Kleopas KA, Kyriakides T. A novel movement disorder of the lower lip. Mov Disord 2004;19:663-704.

9. Ondo W. Lingual dystonia following electrical injury. Mov Disord 1997; 12:253.

10. Tan EK, Jankovic J. Botulinum toxin A in patients with oromandibular dystonia: long-term follow-up. Neurology 1999;53:2102-2107.

11. Charles P, Davis T, Shannon K, Hook M, Warner J. Tongue protrusion dystonia: treatment with botulinum toxin. South Med J 1997;90:522-525.

12. Blitzer A, Brin MF, Fahn S. Botulinum toxin injections for lingual dystonia. Laryngoscope 1991;101:799. 